\title{
INFLUÊNCIA DA SALINIDADE NA TAXA DE EVAPORAÇÃO DA ÁGUA
}

\author{
Djeson M. A. da Costa \\ Professor do Centro Federal de Educação Tecnológica do Rio Grande do Norte,Engenheiro \\ Químico, Licenciado em Química e Mestre em Geociências \\ djeson@cefetrn.br \\ Henio Normando de Souza Melo \\ Professor da Universidade Federal do Rio Grande do Norte, Engenheiro Químico \\ henio@eq.ufrn.br \\ Sebastião R. Ferreira \\ Professor da Universidade Federal do Rio Grande do Norte, Engenheiro Químico \\ ferreira@eq.ufrn.br
}

\begin{abstract}
RESUMO
A alta taxa de evaporação de água, comum em regiões de clima árido e/ou semi-árido, é um fator que afeta significativamente os aspectos qualitativos e quantitativos dos reservatórios de água, principalmente os de superfície. Esse processo, em alguns casos, torna os recursos hídricos disponíveis impróprios para o consumo humano e uso na agropecuária. Este trabalho teve por objetivo analisar os índices evaporativos, em casa de vegetação, de soluções aquosas salinas quando acondicionadas em mini-tanques plásticos. $O$ experimento foi conduzido no Centro Federal de Educação Tecnológica do Rio Grande do Norte, em NatalRN, durante o período compreendido entre 23/07/06 e 23/09/06. Observou-se que a redução na taxa de evaporação em mini-tanque, devido ao aumento na salinidade da água, variou linearmente com a condutividade elétrica, medida em dS. $\mathrm{m}^{-1}$, com r ${ }^{2}=0,999$.
\end{abstract}

Palavras-chave: Taxa de evaporação, salinidade, mini-tanque.

\section{SALINITY INFLUENCE IN THE RATIO WATER EVAPORATION}

\begin{abstract}
The high water evaporation ratio in arid and semi-arid regions is a fact that affects significantly the qualitative and quantitative aspects of water reservoirs, especially those at the surface. This process, in some cases, makes the hydro resources available, improper to human or livestock consumption. This work intends to analyze the evaporative ratio, inside greenhouse, in aqueous saline solutions when contained in plastic mini-tanks. The experiment was carried out at Centro Federal de Educação Tecnológica do Rio Grande do Norte, in Natal-RN, over the period; July 23rd 2006 to September 23th 2006. We observed that decrease in evaporation ratio in mini-tanks, because the increase in the solution salinity, linearly changed with electric conductivity, in dS. $\mathrm{m}^{-1}$, showing $\mathrm{r}^{2}=0,999$.
\end{abstract}

Key-words: Evaporation ratio, salinity, mini-tanks. 


\section{INFLUÊNCIA DA SALINIDADE NA TAXA DE EVAPORACุÃO DA ÁGUA}

\section{INTRODUÇÃO}

A evaporação é um fenômeno que, através dele, átomos ou moléculas no estado líquido absorvem energia suficiente para passar para o estado gasoso. A absorção de energia faz com que as espécies químicas aumentem sua energia cinética tornando suficiente para vencer as forças de atração entre elas, tensão superficial e evapore. Neste caso, o aumento da temperatura eleva o estado de agitação das partículas, aumenta a pressão de vapor e, consequentemente, a evaporação torna-se mais rápida.

O processo da evaporação depende de vários fatores, entre eles os mais significativos são: concentração da substância evaporante, concentração de outras substâncias no ar, temperatura, vazão de ar, umidade relativa do ar, forças intermoleculares, pressão barométrica etc.

A produção de alimentos e vegetais em ambiente protegido tem crescido bastante no Brasil, principalmente nas regiões Sul e Sudeste (Galvani et al., 1998) e atinge aproximadamente 1000 ha., sendo utilizados para o cultivo e a produção de plantas ornamentais, hortaliças e mudas (Oliveira, 1995).

O uso de cobertura plástica para a proteção de ambientes, casa de vegetação, altera alguns dos parâmetros que interferem no processo de evaporação da água, como por exemplo, velocidade do vento, umidade relativa do ar e do solo, temperatura do ar, radiação solar (Galvani et al., 1998). No interior desses ambientes, devido à redução da radiação solar e da velocidade do vento, os conteúdos evaporativos tornam-se menos acentuados (Martins et al., 1999).

As condições meteorológicas afetam o processo da evapotranspiração, seja no meio exterior ou interior à casa de vegetação. Segundo Monteiro et al. (1985), a evaporação no interior de ambientes protegidos atinge aproximadamente de 60 a $80 \%$ dos valores obtidos para o meio externo.

A evapotranspiração de referência ( $\left.\mathrm{Et}_{\mathrm{o}}\right)$ no interior de ambiente protegido é sempre inferior, ficando entre $45 \%$ e $77 \%$ da verificada para o meio exterior (Farias et al., 1994). Existem vários métodos para estimar a $\mathrm{Et}_{\mathrm{o}} \mathrm{e}$, um dos mais comuns utiliza o tanque classe $\mathrm{A}$, desenvolvido pelo Serviço Meteorológico dos Estados Unidos da América (USWB), muito usado também no Brasil (Pereira et al., 1997).

Todos os recursos hídricos superficiais ou subterrâneos possuem sais dissolvidos e sua concentração salina se altera à medida que a água evapora. $\mathrm{O}$ aumento da salinidade afeta o processo evaporativo devido à diminuição da pressão de vapor da solução, em relação ao solvente puro. Portanto, o manejo dos recursos hídricos disponíveis assume um papel importante para a sustentabilidade da produção agropecuária.

Ao adicionar-se água salina, por meio da irrigação, parte é absorvida pelas plantas, outra se infiltra no solo e o restante evapora-se. Os sais dissolvidos na água são precipitados sobre a 
superfície do solo ou na região adjacente, quando ela sofre evaporação, e esse processo pode provocar a salinização do substrato, podendo até torná-lo estéril (Richards, 1993).

A produção agrícola em regiões áridas e semi-áridas depende do abastecimento de água de qualidade adequada. Nas áreas com predominância da agricultura irrigada onde há escassez de água se dá ênfase comumente a métodos que aumentem a qualidade da água (Grattan e Rhoades, 1990). Para melhorar as características dos recursos hídricos disponíveis o país ou a região deve ser capaz de utilizar água de má qualidade. A estratégia de uso dessas águas está em estimar as fontes de água: uma de boa qualidade (não salina) e outra de qualidade inferior (salina) embora não seja necessário que estejam disponíveis durante todo o ano ou toda temporada (Almeida e Gisbert, 2006).

A simples medida da taxa de evaporação, em tanque classe A (EV), já representa grande parte da evapotranspiração potencial da cultura $\left(E T_{\mathrm{P}}\right)$, pois quando a cultura está plenamente desenvolvida, requer uma quantidade de água equivalente a 80 a $90 \%$ da quantidade evaporada de uma superfície livre (Manica, 1973).

Este trabalho teve por objetivo avaliar, em casa de vegetação, a influência da salinidade na taxa de evaporação da água (EV) em mini-tanque, tipo classe $\mathrm{A}$, relacionando-a com a evaporação potencial dos reservatórios superficiais, bastantes utilizados na região Nordeste do Brasil.

\section{MATERIAIS E MÉTODOS}

Este experimento foi realizado em casa de vegetação localizada no Centro Federal de Educação Tecnológica do Rio Grande do Norte (CEFET-RN), na cidade de Natal-RN, situada nas coordenadas geográficas de 5॰48’37,5" latitude sul e 35¹2'14,4” longitude oeste, numa altitude média de $57 \mathrm{~m}$.

Utilizaram-se quatro vasos plásticos (cada um com $491 \mathrm{~cm}^{2}$ de superfície) com soluções salinas (S1, S2, S3 e S4), preparadas em laboratório por meio da adição de cloreto de sódio $(\mathrm{NaCl})$ em água destilada, com condutividade elétrica correspondentes a 0,147; 1,500;3,000 e 4,500 dS. $\mathrm{m}^{-1}$, respectivamente.

Durante o período experimental (23/07/06 a 23/09/06) foram feitas diariamente medidas da umidade relativa do ar (máxima e mínima), com auxílio de um higrômetro digital; da temperatura do ar (máxima e mínima), utilizando termômetro digital; e da massa de água evaporada em cada mini-tanque tipo classe A, com o emprego de uma balança digital. Os valores da massa de água evaporada nos mini-tanques foram calculados pela diferença entre duas medidas consecutivas.

Para que a salinidade da solução, em cada mini-tanque, não fosse submetida a variações muito elevadas em relação aos valores iniciais, diariamente se adicionou a cada mini-tanque o mesmo conteúdo de água evaporada no respectivo período.

Os dados foram estatisticamente submetidos à análise de variância, teste $\mathrm{F}$, ao nível de $5 \%$ de probabilidade. 
A partir dos dados obtidos, diariamente, nos mini-tanques de evaporação reduzida determinou-se a taxa de evaporação acumulada para o período, em função do nível de salinidade da água. Estimou-se, ainda, a evaporação potencial para um reservatório de superfície, com área de $1 \mathrm{~km}^{2}$, a partir da taxa de evaporação no mini-tanque (EV).

\section{RESULTADOS E DISCUSSÕES}

Não foram observadas temperaturas muito altas durante o período experimental, como apresentada na Figura 1A, sendo que a máxima, a mínima e a média para o período, para o interior da casa de vegetação, assumiram valores iguais a $37,6{ }^{\circ} \mathrm{C}, 21,4{ }^{\circ} \mathrm{C}$ e $29,2{ }^{\circ} \mathrm{C}( \pm 1,1)$, respectivamente.

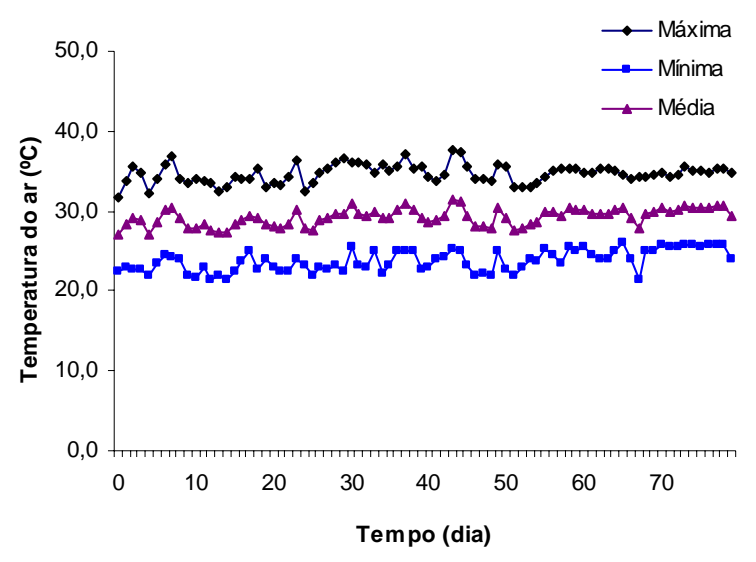

A

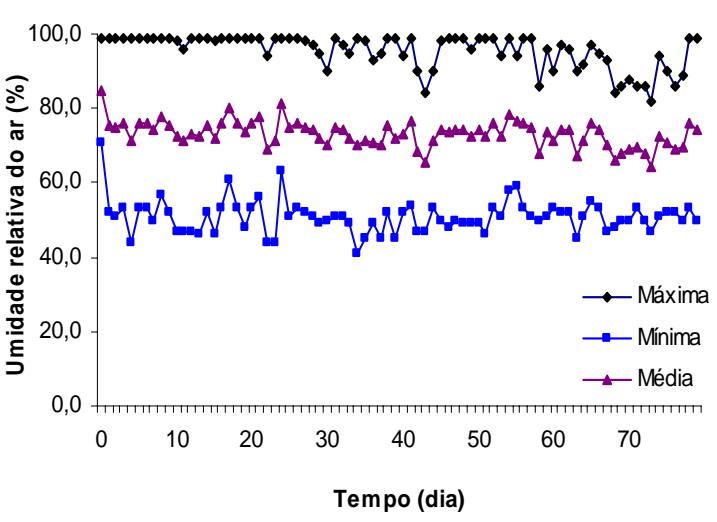

B

Figura 1: Variação diária da temperatura (A) e da umidade relativa do ar (B) no interior da casa de vegetação durante o período experimental.

A umidade relativa máxima e mínima diária do ar no interior da casa de vegetação foi de 99 $\%$ e $41 \%$, respectivamente, com valor médio para o período igual a 73,2 \% $( \pm 3,5)$, como representada na Figura 1B.

Observou-se que as flutuações da temperatura do ar estão sempre associadas às da umidade relativa do ar, ou seja, quando a umidade aumenta ocorre ligeira redução na temperatura.

A dispersão dos dados relativos à temperatura e umidade relativa do ar no interior da casa de vegetação, calculada pelo coeficiente de variação $(\mathrm{CV})$, mostrou-se bastante reduzida, tanto para a temperatura $(\mathrm{CV}=3,66 \%)$ como para a umidade relativa do ar $(\mathrm{CV}=4,75 \%)$.

As magnitudes amenas da temperatura e os valores elevados para a umidade relativa do ar, observados no interior do ambiental protegido, deve-se ao fato de que o período de condução do experimento coincidiu, em parte, com a estação das chuvas.

O aumento da salinidade da água em mini-tanques, no interior da casa de vegetação, proporcionou redução significativa na taxa de evaporação, ao nível de 3,13 \% de probabilidade, como apresentado na Tabela $1 \mathrm{e}$, a análise de regressão mostrou que a 
diminuição do potencial evaporativo variou linearmente com a salinidade da água, medida em termos de condutividade elétrica, como destacado na Figura 2.

Tabela 1: Análise de variância da taxa de evaporação de soluções salina, em ambiente protegido.

\begin{tabular}{lrrrrr}
\hline Fontes de variação & G.L. & S.Q. & Q.M. & F & Probabilidade \\
\hline Entre grupos & 3 & 4,312 & 1,437 & $2,988^{*}$ & 0,0313 \\
Dentro dos grupos & 316 & 152,017 & 0,481 & & \\
\hline Total & 319 & 156,329 & & & \\
\hline
\end{tabular}

G.L.: Grau de liberdade; S. Q.: soma dos quadrados; Q. M.: quadrado médio; F.: fator para análise de variância. " significativo: as médias diferem entre si ao nível de 3,13 \% ( $\left.F_{\text {Critico }}=2,633\right)$.

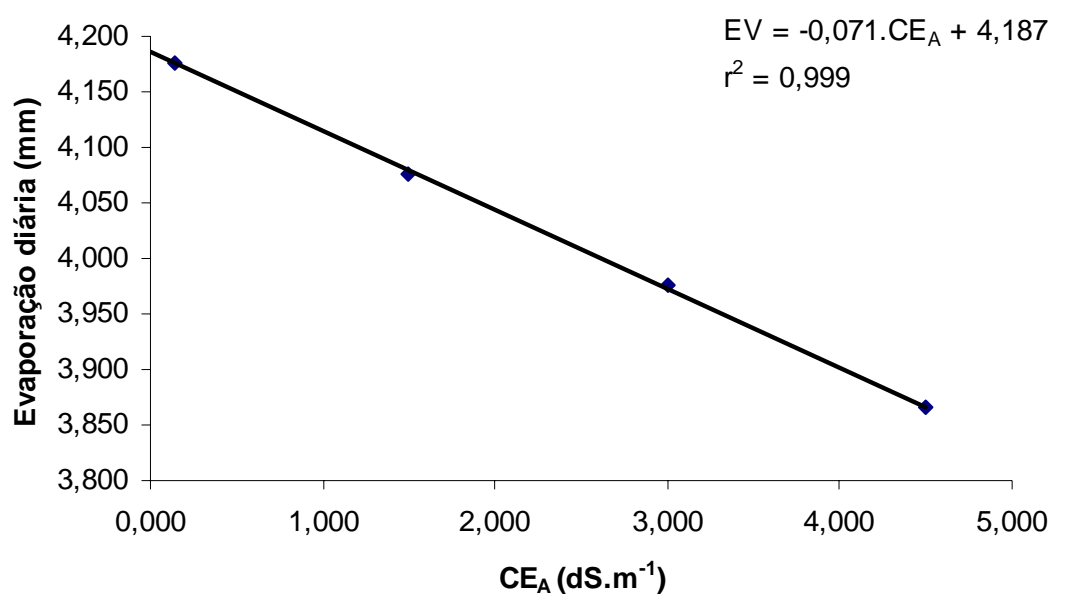

Figura 2: Taxa de evaporação diária em função da salinidade da água, em ambiente protegido.

Verifica-se que o conhecimento da salinidade da água, em termos de condutividade elétrica, é um excelente dado para se estimar com precisão a taxa de evaporação diária em minitanque, no interior de casa de vegetação (EV).

A redução máxima na taxa de evaporação acumulada nos mini-tanques, durante o período experimental, foi de 7,47 \%, da solução S4 em relação à solução $\mathrm{S} 1$, correspondendo a um abaixamento na taxa evaporativa de $5,74 \mathrm{~mm} /\left(\mathrm{dS} . \mathrm{m}^{-1}\right)$, na temporada.

Com base nas informações relativas à redução da taxa de evaporação, constantes no parágrafo anterior, estima-se uma retenção diferencial de aproximadamente $35.670 \mathrm{~m}^{3}$ de água num lago, com superfície de $1 \mathrm{~km}^{2}$ (Farias et al., 1994), quando se compara o potencial evaporativo da solução S4 em relação à solução S1, para um período equivalente ao experimentado. 
Essa economia de água, devido à redução na taxa de evaporação da solução S4 em relação à solução S1, é suficiente para irrigar uma área de aproximadamente 18 ha. de tomateiro (Dalsasso, 1997) ou, então, 20 ha. de meloeiro (Caron e Heldwein, 2000).

\section{CONCLUSÕES}

Nas condições em que os experimentos foram realizados conclui-se que:

1. O aumento da umidade relativa do ar proporcionou redução na temperatura do meio.

2. A taxa de evaporação diária diminui linearmente com o aumento da salinidade da água.

3. O manejo adequado dos reservatórios hídricos de superfície, principalmente em áreas de alta incidência de radiação solar, pode implicar numa maior disponibilidade de água, para uso na agropecuária.

\section{BIBLIOGRAFIA}

1. Almeida, O. A.; Gisbert, J. M. Variación en la calidad del agua de riego en un huerto de cítricos. R. Bras. Eng. Agríc. Ambiental, v.10, n.1, p.64-69, 2006.

2. Caron, B.O.; Heldwein, A.B. Consumo d'água e coeficiente de cultura para o meloeiro cultivado em estufa plástica na primavera. Revista Brasileira de Agrometeorologia, Santa Maria, v. 8, n. 1, p. 19-25, 2000.

3. Dalsasso, L.C.M. Consumo de água e coeficiente de cultura do tomateiro (Lycopersicum esculentum, M.) e do pepineiro (Cucumis sativus, L.) cultivados em estufa plástica. Santa Maria: Universidade Federal de Santa Maria, 1997. 84 p. Dissertação (Mestrado em Agronomia), curso de Pós-graduação em Agronomia/UFSM, 1997.

4. Farias, J.R.B.; Bergamaschi, H.; Martins, S.R. Evapotranspiração no interior de estufas plásticas. Revista Brasileira de Agrometeorologia, Santa Maria, v.2, p.17-22, 1994.

5. Galvani, E.; Dantas, R.T.; Escobedo, J.F.; Klosowski, E.S. Parâmetros meteorológicos em cultura de alface (Lactuca sativa, L.) cultivada em casas de vegetação com orientações leste-oeste, norte-sul e condições externas. Revista Brasileira de Agrometeorologia, Santa Maria, v.6, n.2, p.157-63, 1998.

6. Grattan, S. R.; Rhoades, J. D. Irrigation with saline ground water and drainage water. In: K. K. Tanji (es). Agricultural Salinity Assessment and Management. Manuals and Reports on Engineering Practice n.71, New York: America Society of Civil Engineers ASCE, 1990. Chap. 20, p.432-449.

7. Manica, I. Irrigação em sulcos e sua influência no crescimento e produção da planta matriz de bananeira (Musa Cavendishi Lambert) c.v Nanicão. Piracicaba: ESALQ, 1973. 100p. (Tese - Doutorado em Irrigação e Drenagem).

8. Martins, S.R.; Fernandes, H.S.; Assis, F.N. de; Mendez, M.E.G. Caracterização climática e manejo de ambientes protegidos: a experiência brasileira. Informe Agropecuário, Belo Horizonte, v.20, n.200/201, p.15-23, 1999.

9. Montero, J.I.; Castilla, N.; Gutierrez de Ravé, E.; Bretones, F. Climate under plastic in the Almeria. Acta Horticulturae, The Hague, v.170, p.227-34, 1985.

10. Oliveira, M.R.V. de. O emprego de casas de vegetação no Brasil: vantagens e desvantagens. Pesquisa Agropecuária Brasileira, Brasília, v.30, n.8, p.1049-60, 1995. 
11. Pereira. A.R.; Villa Nova, N.A.; Sediyama, G.C. Evapo(transpi)ração. Piracicaba: FEALQ, 1997. 183 p.

12. Richards, L. A. Diagnóstico y recuperación de suelos salinos y sódicos. Personal de Laboratorio de Salinidad de EE.UU. 6.ed. 7. reimpresión. México: Editorial LIMUSA. 1993. 176p. 